\title{
A novel observer algorithm of voltages across capacitors based on the higher sliding mode control: application to multi-cells converter
}

\section{Mohamed Redha Skender and Abdelhalim Tlemçani}

Laboratoire de Recherche en Electrotechnique et en Automatique,

Universite Yahia Farès de Medea, Algeria

Email: skender_mohamed@yahoo.fr

Email: atlemcani@asso-enp.org

\section{Hassan Nouri*}

Power Systems, Electronics and Control Research Group,

University of the West of England,

Bristol, BS16 1QY, UK

Email: hassan.nouri@uwe.ac.uk

*Corresponding author

\begin{abstract}
In this paper, advanced automatic sliding mode observers are applied to multi-cells converter. The technique facilitates the generation of the voltages image across capacitors of the converter simply by measuring the current. Also, the technique possesses the finite time convergence error. The validity of the controller is demonstrated through simulation results in both the mentioned properties of the technique. Furthermore, a topology with a higher order sliding mode observer for multi-cell converters applications are proposed.
\end{abstract}

Keywords: series multi-cells converter; higher order sliding mode; observer; super twisting.

Reference to this paper should be made as follows: Skender, M.R., Tlemçani, A. and Nouri, H. (2017) 'A novel observer algorithm of voltages across capacitors based on the higher sliding mode control: application to multi-cells converter', Int. J. Modelling, Identification and Control, Vol. 27, No. 2, pp.136-145.

Biographical notes: Mohamed Redha Skender is a $\mathrm{PhD}$ candidate and holds a position as a Laboratory Engineer in Electrical Engineering at the University of Medea, Algeria. He received his Engineering degree in Electrical Engineering from the University of Medea in 1994 and Master degree from the University of Medea in 2011. His research interests include power electronics, electrical drives, nonlinear control, computer control and industrial automation.

Abdelhalim Tlemçani received his BSc and MSc in Power Electronics and PhD in Electrical Engineering from the National Polytechnic School of Algiers, Algeria, in 1997, 1999, and 2007, respectively. In 2002, he was a Lecturer and researcher with the Department of Electrical Engineering, Université Docteur Yahia Farès de Médéa, Medea, Algeria, where he is currently a Professor. He is the Director of the Control and Power Electronics Research Group. His research interests include power electronics, electrical drives, robust and nonlinear control, and fuzzy systems.

Hassan Nouri received his $\mathrm{BSc}, \mathrm{MSc}$, and $\mathrm{PhD}$ degrees in Electrical and Electronic Engineering from the universities of Nottingham, Strathclyde and Plymouth, UK. He is currently a Reader in Electrical Power and Energy and Leader of the Power Systems, Electronics and Control Research Laboratory in the Department of Engineering Design and Mathematics at UWE, Bristol, UK. His extensive research work covers power systems analysis, power system modeling, power electronics, electric arc modelling, fault locations, power quality, control and electrical technology.

This paper is a revised and expanded version of a paper entitled 'Sliding mode observer control founded to series multi-cell converter' presented at 4th International Conference on Electrical Engineering (ICEE), Boumerdes, Algeria, 13-15 December 2015. 


\section{Introduction}

Power electronics is currently present in many industries given its very broad scope of application, especially in high power, owing to the evolution of the semiconductor components power and new topologies energy conversion. Among these topologies, multi-cells converters, which appeared in the early ' $90 \mathrm{~s}$ (Dhanalakshmi et al., 2015; Meynard et al., 2002), are based on the series association of elementary switching cells. Associations of elementary switching cells are best technical solutions that are now becoming very competitive. The switch control allows to cancel the harmonics at the switching frequency and reduce the ripple voltage chopped by using different voltage levels, thus to reduce switching losses. Anyway, knowledge of the capacitor voltages is always necessary.

Conventional control laws give good results in the case of linear systems with constant parameters. For the parameters of nonlinear system or with non-constant conventional control laws may be insufficient because they are not robust, particularly when precision requirements and other dynamic characteristics of the system are strict. It is necessary to use control laws insensitive to changes in parameters and nonlinear disturbances. Order to maintain a correct operation of the converter over time, the control device shall ensure the regulation voltages of capacitors. Thus, the regulation allows one hand to evenly distribute the constraints on each switch, and secondly to maintain the same characteristics viewpoint levels of output voltage.

Recently invented the higher order sliding mode generalise the sliding idea which is the basis of this method, the action is based on the higher order time derivative for the deflection of constraint system (sliding surface) instead of influence on the first derivative of the deviation as it occurs in simple order sliding mode. While keeping the main advantages of the original approach, at the same time, they completely remove the chattering (Ghanes et al., 2009; Kolsi Gdoura et al., 2015).

This command sliding mode requires use of sensors floating voltages whose number increases with the number of cells. In addition, one of the first indisputable successes of the automatic was proposing state observers as substitutes for sensors often expensive or not reliable enough, thus reducing the cost and the bulk of the industrial plant (Zhen et al., 2014).

Furthermore, the normal operation of the series p-cell converter is obtained when the voltages are $v_{c i}=i E / p, i=1$, $\ldots, p-1$ ( $E$ is the input source). These voltages are generated when a suitable control of switches is applied in order to obtain a specific value. The control inserted of the switches allows cancelling the harmonics at the switching frequency and reducing the ripple of the chopped voltage. However, these properties are lost if the voltages of these capacitors drift. On the other hand, if a specific control is desired, it is advisable to measure these voltages in order to implement it. But, it is not easy because extra sensors are necessary to measure these voltages, then it increases the cost. For this reason, it should be avoided and the estimation of these voltages becomes an attractive and economic option. It is for such reason that an original method to eliminate such sensors is the use of observers. From control theory point of view, an observer is considered as a software sensor used to estimate the unmeasurable variables of a system.

On the other hand, several approaches have been considered to develop new methods of control and observation of the p-cell converter. Initially, models have been developed to describe their instantaneous behaviours (Donzel, 2000), harmonic or averaging (Bensaid et al., 1999). These various models were used as the base for the development of control laws in open loop (Tachon et al., 1997) and in closed-loop (Bhave et al., 2015; Bethoux et al., 2003).

Until now, all these p-cell converters are driven successfully, by means of a fix frequency modulator based on pulse width modulation (PWM). Current control algorithms do not take into account the fact that any power converter is a discrete and discontinuous plant, or, at least a hybrid one. Nevertheless, the profitable skill of PWM technique is to ensure a well-known steady state behaviour, which is 'optimal' for the electric load with respect to harmonic attenuation. Furthermore, some representations of the p-cell converter considered complex models and need to be discretised in order to design a discrete observer to be implemented.

Then, in all proposed methods a considerable number of feedback signals are required which are associated with extra cost of sensing devices. To reduce the cost of sensors, a methodology to estimate the voltages in the capacitors is necessary.

In Bestle and Zeitz (1983) and Krener and Isidori (1983), the observer canonical form consisting of a linear output map and linear dynamics driven by a nonlinear output injection is used. The resulting observer has exactly linear error dynamics, i.e., nonlinearities are compensated exactly. The approaches suggested in Gauthier et al. (1992), Dalla Mora et al. (1997) and Busawon et al. (1998) rely on the observability canonical form, which has significantly weaker existence conditions than the observer canonical form. In the observability canonical form, the observer is designed by a high-gain technique with a constant observer gain, i.e., the nonlinearities are not compensated but dominated by a linear part. For an implementation of the observer in the original coordinates one gets a Luenberger-like observer with a possibly nonlinear gain vector field (Röbenack, 2007; Chen and Zhai, 2007). In the last decade, new approaches have been developed for nonlinear systems that are not uniformly observable. Several approaches use Kalman-like decompositions (Röbenack, 2007).

In this article, we develop an observer for p-cell chopper based on an instantaneous model describing the dynamical behaviour of the p-cell converter using higher order sliding mode. This model is constructed in order to design an observer estimating each flying capacitor voltage. The proposed observer design is based on the class of nonlinear systems which can be written in the form of affine state 
systems, for which the problem of state observer design has been studied. This class of observers is based on the excitation condition in order to guarantee its convergence.

The paper is organised as follows. In Section 2, the position of the problem is identified. In Sections 3 and 4, the description model of the multi cells converter with three cells is discussed and implementation of the higher order sliding mode control of the converter is described respectively. Finally, in Section 5, a higher order sliding mode observers for a series multi cells converter with all its mathematical algorithms is discussed.

\section{Position of the problem}

In this section, we consider a nonlinear system whose dynamics are described by the differential system:

$$
\begin{aligned}
& \dot{x}=f(t, x, u) \\
& s=s(t, x) \in \Re \\
& u=u(t, x) \in \Re
\end{aligned}
$$

where

$x=\left[x_{1}, \ldots \ldots, x_{n}\right]^{T} \in X$ represents the state vector, $\mathrm{X} \subset \Re^{n}$, $X$ is a differentiable manifold or an open subset of $\Re^{n}$.

$u$ represents the command

$f \quad$ is assumed sufficiently differentiable function, but known so uncertain

$t$ is the time.

The system $\dot{x}=f(t, x, u)$ includes those of the form (Bhave et al., 2015):

$$
\dot{x}=f(x)+g(x) u,
$$

The problem is always to force the trajectories of the system to evolve on the sliding surface

$$
S=\{x \in X: s(t, x)=0\}
$$

Being a real-valued function sufficiently differentiable such that its $(r-1)$ first derivatives with respect to time are functions of the $x$ state (which means they do not contain any discontinuity.) defines a submanifold of $(n-1)$ dimension called sliding surface or switching.

The $s(t, x)$ function is called a sliding function or switching function. It separates the state space in two separate parts $s(t, x)>0$ and $s(t, x)<0$.

\section{Description of a series multi-cells converter}

This section describes the design of the series multi cells converter for a four-level converter supplying an inductive load (Figure 1).

The instantaneous state model of a chopper with three cells is expressed as follows (Ben Said et al., 2013; Liu et al., 2013):

$$
\left\{\begin{array}{l}
\dot{x}_{1}=f_{1}(x, u)=\delta_{1} a_{1} x_{3} \\
\dot{x}_{2}=f_{2}(x, u)=\delta_{2} a_{3} x_{3} \\
\dot{x}_{3}=f_{3}(x, u)=-b_{0} x_{3}-b_{1}\left(\delta_{1} x_{1}+\delta_{2} x_{2}-u_{3} E\right) \\
y=h(x)=x_{3}
\end{array}\right.
$$

$\left(x_{1}, x_{2}, x_{3}\right)^{T}=\left(V_{c 1}, V_{c 2}, I_{c h}\right)^{T}$ is the state vector as: $x \in \mathfrak{R}^{3}$

$y=h(x) \quad$ is the measurement vector $y \in \Re$

$\left(u_{1}, u_{2}, u_{3}\right)$ are controls switches.

$$
a_{1}=a_{2}=\frac{1}{C}, b_{0}=\frac{R}{L}, b_{1}=\frac{1}{L}, \delta_{1}=u_{2}-u_{1},
$$

$\delta_{2}=u_{3}-u_{2}$ are coefficients.

Figure 1 Chopper three cells connected to a $R L$ load

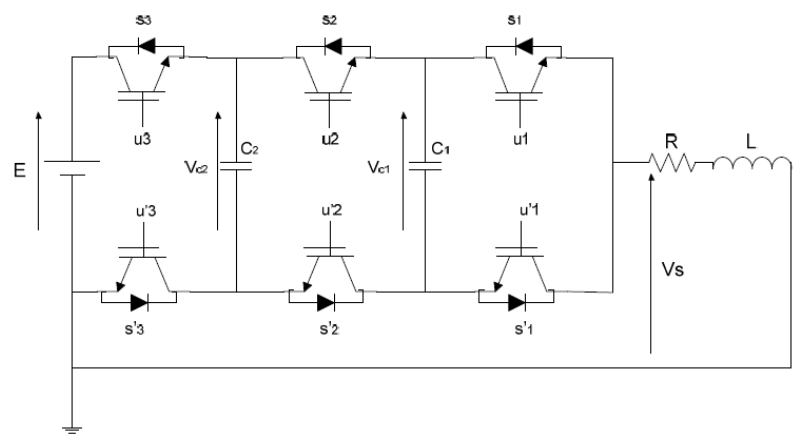

\section{A higher order sliding mode control structure}

\subsection{Calculation of switching functions}

The multi-cells converter as it was defined and modelled is a nonlinear multi-input variable structure system. Define a sliding mode control of such a system is equivalent to setting $p$ sliding surfaces (each switching cell is an $s_{i}(x)=0$ surface of $n-1$ dimensions) associated with that switching law properly allow the stability and the existence of a sliding regime on the intersection of all surfaces (Benzineb et al., 2011). The trajectory evolution of the system with respect to the position of the surface determines the state of the switches.

$$
\dot{x}=f(x)+\sum_{i=1}^{p} g_{i}(x) u_{i}=f(x)+G(x) U
$$

By a change of variable, we can define the dynamics of the converter according to the error:

$$
\Delta \dot{x}=f(x)+G(x) U_{e q}+G(x) \Delta U
$$

where

$$
\Delta U=-U-U_{e q}
$$

with $U_{e q}$ : the equivalent command to keep the system in steady state.

The choice of the Lyapunov function is based on the simplicity of control law implementation. Other choices of the Lyapunov function can be considered and ensure better 
performance by imposing a dynamic or minimising a quadratic criterion (Meradi et al., 2013). We consider following Lyapunov function:

$$
V(\Delta x)=\frac{1}{2} \Delta x^{T} \cdot Q \cdot \Delta x
$$

And its time derivative of $V$ is:

$$
\dot{V}(\Delta x)=\Delta x^{T} \cdot Q \cdot \Delta \dot{x}
$$

where

$$
\begin{aligned}
& Q=\operatorname{diag}\left(C_{1}, C_{2}, \ldots \ldots \ldots, C_{P-1} L\right) \\
& \Delta x^{T}=x-X_{\text {ref }} \\
& =\left[v_{c 1}-\frac{E}{p} v_{c 2}-\frac{2 E}{p} \cdots v_{c p-1}-\frac{(p-1) E}{p} i-I_{r e f}\right]^{T} \\
& X_{\text {ref }}=\left[\begin{array}{lllll}
\frac{E}{p} & \frac{2 E}{p} & \cdots & \frac{(p-1) E}{p} & I_{\text {ref }}
\end{array}\right]
\end{aligned}
$$

After demonstration, we then choose $p$ switching functions:

$$
s_{i}(x)=-\Delta x^{T} \cdot Q \cdot g_{i}(x)
$$

As a result, the state of the switches is a function of $p$ switching functions. The state of each switch is defined by:

$$
u_{i}= \begin{cases}1 & \text { if } s_{i}(x)>0 \\ 0 & \text { if } s_{i}(x)<0\end{cases}
$$

We can now define the control laws that ensure stability of the $X_{\text {ref }}$ equilibrium point using the results of the previous section for a three cells converter.

$s_{1}, s_{2}$ and $s_{3}$ switching functions are obtained from the following expressions:

$$
\left\{\begin{array}{l}
s_{1}(x)=-\Delta x^{T} \cdot Q \cdot g_{1}(x)=I_{r e f} v_{c 1}-\frac{E}{3} I_{c h} \\
s_{2}(x)=-\Delta x^{T} \cdot Q \cdot g_{2}(x)=I_{e r f}\left(v_{c 2}-v_{c 1}\right)-\frac{E}{3} I_{c h} \\
s_{3}(x)=-\Delta x^{T} \cdot Q \cdot g_{3}(x)=I_{r e f}\left(E-v_{c 2}\right)-\frac{E}{3} I_{c h}
\end{array}\right.
$$

Here, $I_{c h}$ represents the load current and after some transformations, these functions in homogeneous form can be rewritten as:

$$
\left\{\begin{array}{l}
s_{1}(x)=\frac{3 I_{r e f}}{E} v_{c 1}-I_{c h} \\
s_{2}(x)=\frac{3 I_{r e e f}}{E}\left(v_{c 2}-v_{c 1}\right)-I_{c h} \\
s_{3}(x)=\frac{3 I_{r e f}}{E}\left(E-v_{c 2}\right)-I_{c h}
\end{array}\right.
$$

\subsection{Super twisting algorithm}

The algorithm convergence is also governed by the rotation around the origin of the phase diagram. The super twisting $u(t)$ control law consists of two parts. The first $u_{1}$ is defined by its derivative with respect to time, while the second $u_{2}$ is given by the sliding variable of continuous function (Meradi et al., 2013):

$$
\begin{aligned}
& u=u_{1}+u_{2} \\
& \dot{u}_{1}=\left\{\begin{array}{cc}
-u & \text { si }|u|>1 \\
-W \operatorname{sign}(s) & \text { si }|u| \leq 1
\end{array}\right. \\
& u_{2}=\left\{\begin{array}{cc}
-\lambda_{1}\left|s_{0}\right|^{p 1} \operatorname{sign}(s) & \text { si }|s|>s_{0} \\
\lambda_{1}|s|^{p 1} \operatorname{sign}(s) & \text { si }|s| \leq s_{0}
\end{array}\right.
\end{aligned}
$$

This controller can be simplified if the control system is linearly dependent on the control and the law control is given by:

$$
\left\{\begin{array}{l}
u=\lambda|s|^{\frac{1}{2}} \operatorname{sign}(s)+v \\
\dot{v}=-W \operatorname{sign}(s)
\end{array}\right.
$$

The interest of this algorithm is that it very robust; it does not need information about the derivative of $s$. Because of this characteristic can reduce the number of sensors in the system, and the calculation time.

\subsection{Super twisting control of a three cells choppers}

The proposed control strategy is based on the super twisting algorithm. In this context, we consider the sliding surfaces with the $s_{1}, s_{2}$ and $s_{3}$ switching functions obtained in (8).

The relative degree for that subsystem $(r=n=1)$. When $r=1$, the law control is the super twisting law.

The duty ratios are calculated by the following law control:

$$
\left\{\begin{array}{l}
\alpha_{i}=\lambda_{i}\left|s_{i}\right|^{\frac{1}{2}} \operatorname{sign}\left(s_{i}\right)+v_{i} \\
\dot{v}_{i}=-W_{i} \operatorname{sign}\left(s_{i}\right) \\
\text { avec }: i=1,2,3
\end{array}\right.
$$

\subsection{Simulation results}

We will validate the dynamic performance of the law control using a simulation for a converter (chopper and inverter) with the following characteristics:

$$
\left\{\begin{array}{l}
C_{1}=C_{2}-40 \mu F \\
L=0.5 m H \\
R=10 \Omega
\end{array}\right.
$$

The following command sequence is used: $80 \mathrm{~A}$.

The voltage source is $1,500 \mathrm{~V}$, the reference current is

At $t=0.25 \mathrm{~s}$, the source voltage is set to $E=1,800 \mathrm{~V}$.

We use a regulator with the following parameters:

$$
\left\{\begin{array}{l}
\lambda_{1}=\lambda_{2}=\lambda_{3}=20 \\
W_{1}=W_{2}=W_{3}=10
\end{array}\right.
$$


Our objective is to regulate the voltages $v_{c 1}$ and $v_{c 2}$ of capacitors their references $\frac{E}{3}$ and $\frac{2 E}{3}$ and the load current must reach its reference value $I_{\text {ref }}=80 \mathrm{~A}$.

A value $I_{r e f}$ reference current is independent of the load.

\subsubsection{Super twisting control of a three cells chopper}

The control scheme is shown in Figure 2.

Figure 2 Structure of super twisting control of chopper (see online version for colours)

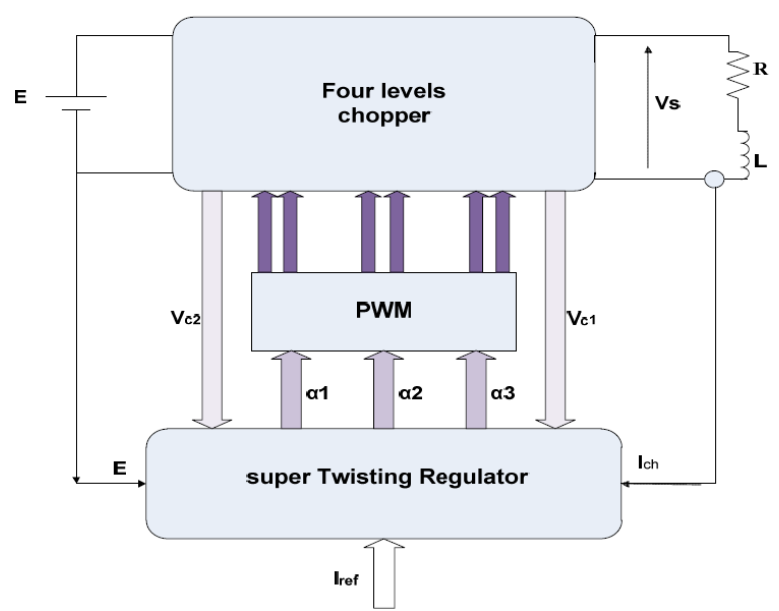

We obtained the following results.

Figure 3 Evolution of the $v_{c 1}$ and $v_{c 2}$ floating voltage (see online version for colours)
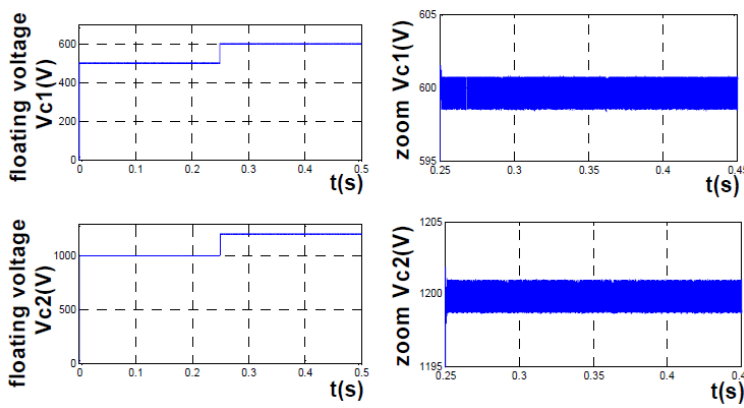

Figure 3 shows the evolution of floating voltages, voltages quickly to assess steady state, before and after the change of the reference voltage at time $t=0.25 \mathrm{~s}$, we find that the two voltages $v_{c 1}$ and $v_{c 2}$ respectively to stabilise at $\frac{E}{3}$ and $\frac{2 E}{3}$, and the phenomenon of chatter is gone.

Figure 4 Evolution of the $I_{c h}$ load current (see online version for colours)
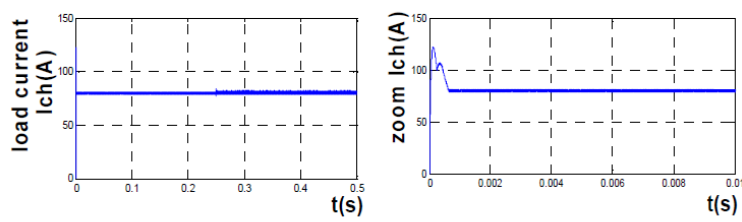

Figure 4 represents the evolution of the $i_{c h}$ load current, it is clear that current enslaves a reference value. And after the zoom, the current has exceeded due to speed of response that characterises the law of the synthesised sliding mode control.

Note that the two voltages $v_{c 1}$ and $v_{c 2}$ and load current $i_{c h}$ follow their references.

The evolution of the output voltage is represented in Figure 5. By zooming Figure 5, we can clearly notice the presence of four voltage levels namely $E, \frac{2 E}{3}, \frac{E}{3}$ and 0 regardless of the changes to the sequence reference voltage.

Figure 5 Evolution of the $V_{s}$ output voltage (see online version for colours)
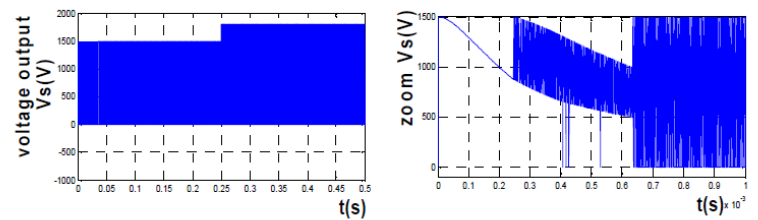

Figure 6 Evolution of the sliding surfaces (see online version for colours)
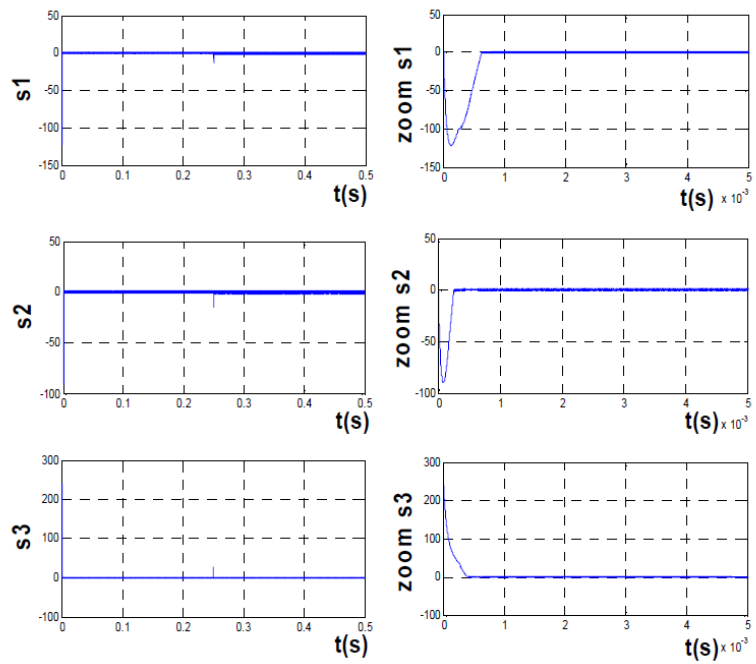

Figure 6 shows the evolution of three switching surfaces, which is around 0 . When there is a variation in the reference voltage, the sliding surfaces move away from the value 0 but return very quickly to zero in order to cancel the command.

\subsubsection{Super twisting control of a three cells three- phased inverter}

The control scheme is shown in Figure 7.

With the same parameters as for the chopper, the sliding mode command is applied on a three phase inverter.

We use a regulator with the following parameters:

$\left\{\begin{array}{l}\lambda_{1}=\lambda_{2}=\lambda_{3}=152 \\ W_{1}=W_{2}=W_{3}=5\end{array}\right.$

The shape of the reference current is: 


$$
I_{r e f}=80 \sin \left(100 \pi t+(k-1) \frac{2 \pi}{3}\right), k=1,2,3
$$

We obtained the following results:

Figure 7 Structure of super twisting control of inverter (see online version for colours)

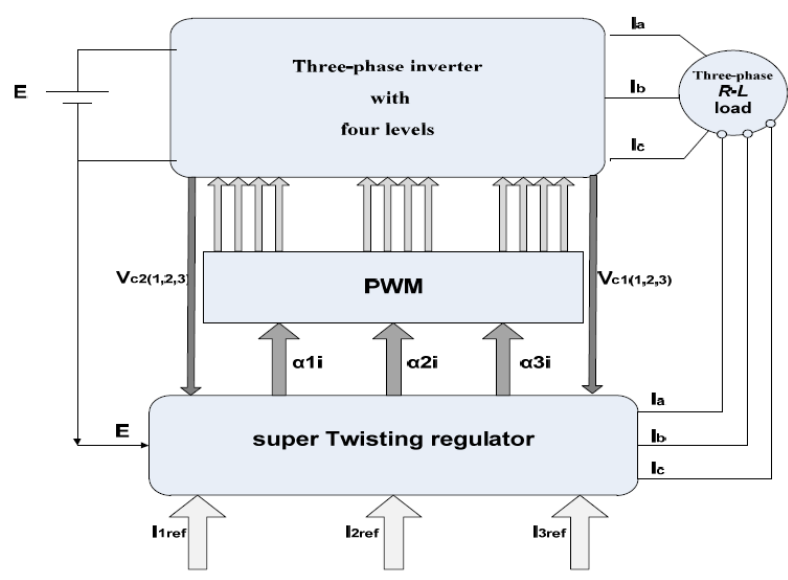

Figure 8 Evolution of the $v_{c 1}$ and $v_{c 2}$ floating voltage of the first arm (see online version for colours)
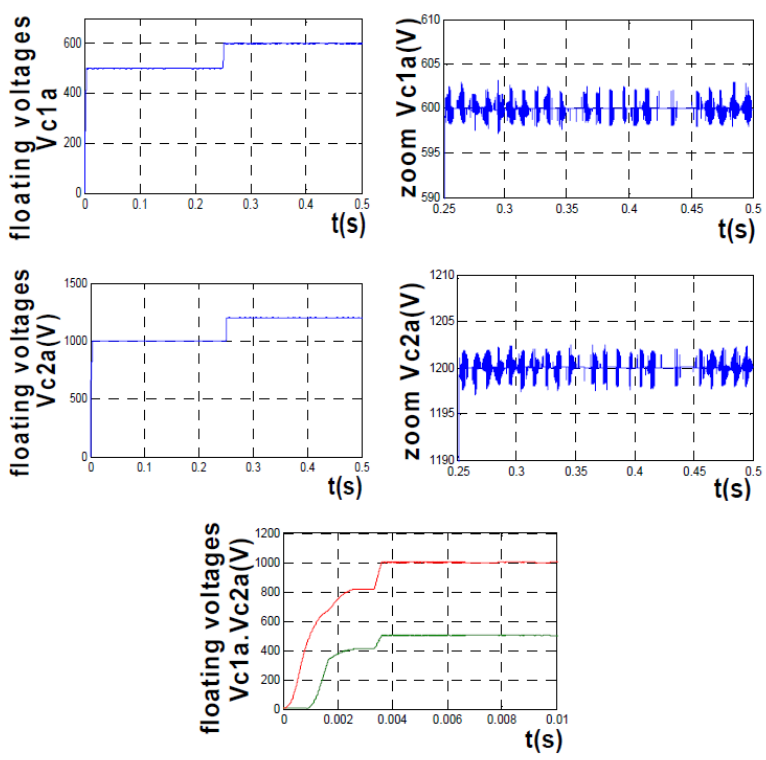

Figure 8 shows the evolution of floating voltages of the arms. These voltages settle at the reference value $\frac{E}{3}$ and $\frac{2 E}{3}$.

Figure 9 Evolution of the $V_{a}$ output voltage of the first arm (see online version for colours)
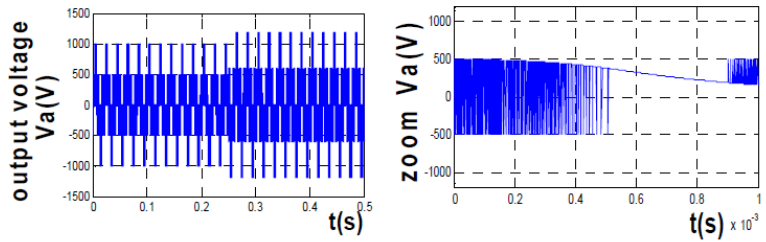

Figure 9 shows the evolution of the $V_{a}$ output voltage of the first arm which is found to have a range of $\frac{2 E}{3}$ and $-\frac{2 E}{3}$.

Figure 10 Evolution of the load current from the charging current $I_{c h}$ in the first arm (see online version for colours)

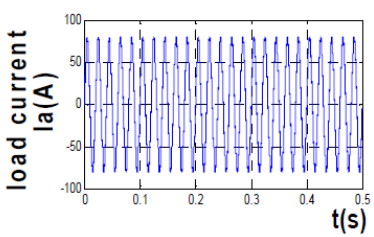

Figure 10 shows the evolution of the load current which is a sinusoidal current and perfectly follows its reference.

\section{A higher order sliding mode observers of a series multi-cells converter}

An observer or state estimator, or software sensor, is a knowledge-based model describing the behaviour of the system and using measurements acquired in the process to reconstruct the missing measures algorithm (Erickson and Maksimovic, 2001).

Now with the advent of multi-cell converters, the number of variables is more important to know which is proportional to the number of cells switching. An observer floating voltages is, in this case, totally justifiable, since it eliminates sensors, thereby reducing the cost and space requirement of the installation.

\subsection{Operating principles of the observer}

In all sliding mode control, the principle of sliding mode observer is to force, using discontinuous functions, the dynamics of ' $n$ ' order system to converged to a variety of ' $n$ - $p$ ' dimensions known as sliding surface ( $p$ is the dimension of the measurement vector).

The attractiveness of this surface is ensured by conditions called sliding conditions. If these conditions are satisfied, the system converges to the sliding surface and $y$ evolves according to a dynamic ' $n-p$ ' order.

In the case of sliding mode observer, the dynamics involved are those errors $\tilde{x}=x-\hat{x}$ of observation, from their $\tilde{x}(0)$ initial values, these errors converging to the equilibrium values in two phases (Meradi et al., 2013).

In the first phase, the trajectories of these errors are forced to move towards the sliding surface where the error between the output of the observer and the $\tilde{y}=y-\hat{y}$ real output system is zero. This step is referred to as the reaching mode and it is highly dynamic (Kaur and Janardhanan, 2014).

The second phase is called a sliding mode where the trajectory error of observation slides on the sliding surface defined by $\tilde{y}=0$ with a dynamic imposed to cancel the rest of the error. 


\subsection{Observer synthesis steps}

The different synthesis steps of the observer sliding mode are known and clearly identified by Slotine et al. (1987). These are recalled below.

Consider a nonlinear $n$ order state affine system of:

$$
\left\{\begin{array}{l}
\dot{x}=f(x, u) \\
y=h(x)
\end{array}\right.
$$

where $x \in \Re^{n}$ is the state vector and $y \in \Re^{n}$ is the measured output vector of the system. The sliding mode observer of such a system is defined with the following structure:

$$
\dot{\hat{x}}=f(\hat{x}, u)-\Lambda \Gamma_{s}
$$

where

$\hat{x} \in \Re^{n}$ is the estimate of the $\mathrm{x}$ state vector

$u$ is the control vector

$f(\hat{x}, u)$ is the estimate of the $f(x, u)$ function with $\hat{x}_{j, j=1, \ldots, n}$

$\Lambda$ is an $n \times p$ matrix containing the observer patches gains

$\Gamma_{s}$ is the $p \times 1$ dimension vector defined as

$$
\Gamma_{s}=\left[\operatorname{sign}\left(\tilde{y}_{1}\right), \operatorname{sign}\left(\tilde{y}_{2}\right), \ldots, \operatorname{sign}\left(\tilde{y}_{p}\right)\right]^{T}
$$

With $\tilde{y}_{i}=\hat{y}_{i}-y_{i}$ and $i=1, \ldots, p$.

Let $\tilde{y}=\hat{y}-y$ the relating vector of the output observation errors and $\tilde{x}=\hat{x}-x$ the state vector.

Then the sliding surface is defined by: $s=\tilde{y}=\hat{y}-y=0$.

Hence, the observation dynamic errors:

$$
\dot{\tilde{x}}=\dot{\hat{x}}-\dot{x}=\Delta f-\Lambda \Gamma_{s}
$$

With:

$$
\Delta f=f(\hat{x}, u)-f(x, u)
$$

Figure 11 shows the block diagram of the observer sliding mode.

Figure 11 The block diagram of the observer sliding mode (see online version for colours)

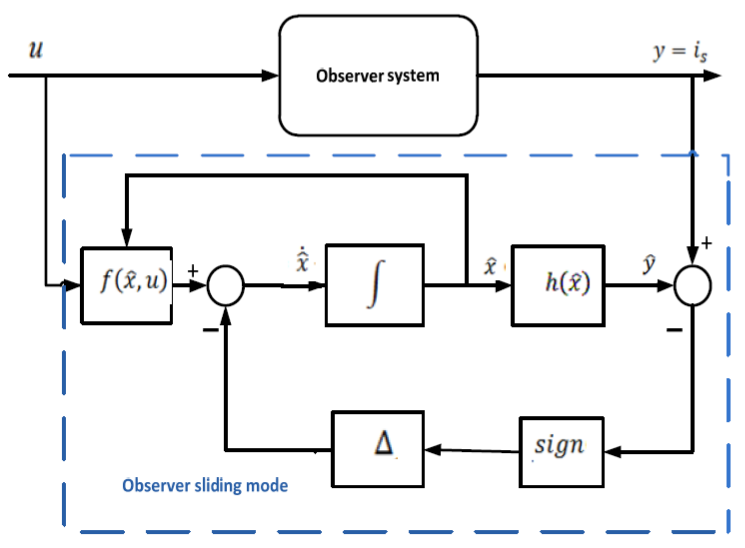

The sliding surface, allowing the synthesis of a sliding mode observer, must satisfy the condition of attractiveness $s . \dot{s}<0$ and the invariance condition:

$$
\left\{\begin{array}{l}
\dot{s}(x)=0 \\
s(x)=0
\end{array}\right.
$$

The attractiveness condition is ensured if the $V(s)=\frac{1}{2} s^{T} . s$ Lyapunov function checks $\dot{V}(x)<0$, when $s \neq 0$, and invariance condition is satisfied using the $\Lambda$ correction terms of (14).

\subsection{Super twisting observer}

The second order sliding mode observer also known as 'super twisting algorithm' (Bhave et al., 2015). In this type of observer the dynamics of the observation error shows that the trajectories of the system evolve after a finite time to a sliding surface and equivalent dynamics on the surface provides the estimate time directly, without recourse to any filter (disadvantage of sliding mode of order 1) through the output of the injection.

The advantage of using sliding mode observers, particularly those second order (super twisting algorithm) is the generation of the voltages image across capacitors of the converter simply by measuring the current and the inherent finite time convergence error.

\subsection{A higher order sliding mode observer of a three cells chopper}

This section describes the design of the second order sliding mode observer for a four-level converter supplying a load $R L$ (Figure 12).

Figure 12 Control diagram of floating voltages with second order sliding mode observer (see online version for colours)

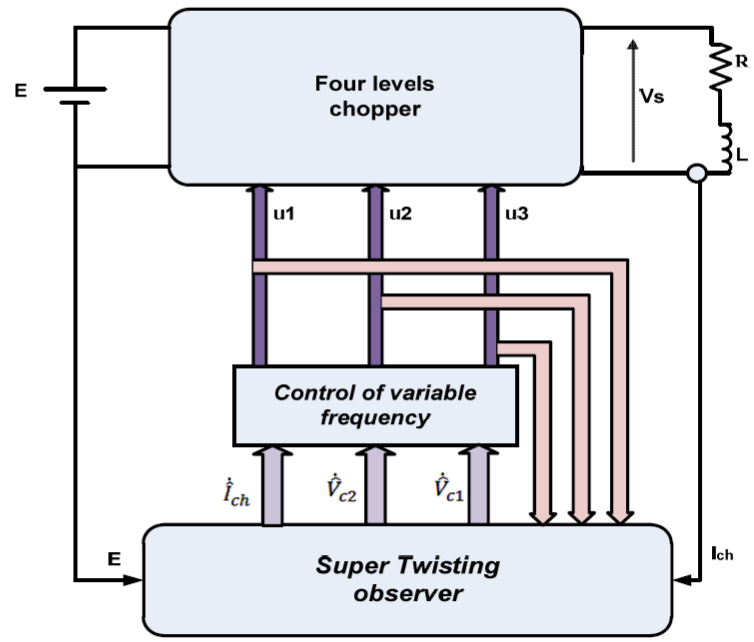


Figure 12 shows the diagram of the sliding mode control with observer floating voltages based on super twisting algorithm.

\subsubsection{The equations of the sliding mode observer}

Applying the theory of the sliding mode observer of (14) for the three-cell converter of (3), we deduce the sliding mode observer to three cells of the converter, where

$\hat{x} \in \mathfrak{R}^{3}$ is the estimated state $x$ vector.

$$
\hat{a}_{1}=\hat{a}_{2}=\frac{1}{\hat{c}}, \hat{b}_{0}=\frac{\hat{R}}{\hat{L}}, \hat{b}_{1}=\frac{1}{\hat{L}}
$$

where $\hat{R}, \hat{L}, \hat{C}$ are the estimated values for passive circuits $f(\hat{x}, u)$ is the estimated model of $f(x, u)$ with $\hat{x}_{j=1,2,3}$

$\Lambda$ is the gain matrix $(3 \times 1)$ specified by the criterion that will be given later

$\operatorname{sign}(s)$ is order vector $p \times 1=1 \times 1$ since $x_{3}=I_{c h}$ is the only measured state.

$s$ is the sliding surface defined as follows:

$s=\hat{x}_{3}-x_{3}=\tilde{x}_{3}$

The dynamic $\dot{\tilde{x}}$ errors observation is then defined as follows:

$$
\left\{\begin{array}{l}
\dot{\hat{x}}_{1}=f_{1}(\hat{x}, u)-z_{1 e}=\delta_{1} \hat{a}_{1} \hat{x}_{3}-z_{1 e} \\
\dot{\hat{x}}_{2}=f_{2}(\hat{x}, u)-z_{2 e}=\delta_{2} \hat{a}_{2} x_{3}-z_{2 e} \\
\dot{\hat{x}}_{3}=f_{3}(\hat{x}, u)-z_{3 e}=-\tilde{b}_{0} \hat{x}_{3}-\tilde{b}_{1}\left(\delta_{1} \tilde{x}_{1}+\delta_{2} \tilde{x}_{2}-u_{3} E\right)-z_{3 e}
\end{array}\right.
$$

$z_{1 e}, z_{2 e}$ and $z_{3 e}$ are correction factors based on the algorithm super twisting. They are given by the formulas:

$$
\left\{\begin{array}{l}
z_{1 e}=\Lambda_{1} \operatorname{sign}(s) \\
z_{2 e}=\Lambda_{2} \operatorname{sign}(s) \\
z_{3 e}=\Lambda_{3}|s|^{1 / 2} \operatorname{sign}(s)
\end{array}\right.
$$

The dynamic observation $\dot{\tilde{x}}$ errors are then defined as follows:

$$
\left\{\begin{aligned}
\dot{\tilde{x}}_{1}=\Delta f_{1}-\Lambda_{1} \operatorname{sign}\left(\tilde{x}_{3}\right)=\delta_{1}\left(\tilde{a}_{1} \tilde{x}_{3}\right)-\Lambda_{1} \operatorname{sign}\left(\tilde{x}_{3}\right) \\
\dot{\tilde{x}}_{2}=\Delta f_{2}-\Lambda_{2} \operatorname{sign}\left(\tilde{x}_{3}\right)=\delta_{2}\left(\tilde{a}_{2} \tilde{x}_{3}\right)-\Lambda_{2} \operatorname{sign}\left(\tilde{x}_{3}\right) \\
\dot{\tilde{x}}_{3}=\Delta f_{3}-\Lambda_{3}\left|\tilde{x}_{3}\right|^{1 / 2} \operatorname{sign}\left(\tilde{x}_{3}\right)=-\tilde{b}_{0} \tilde{x}_{3} \\
\quad-\tilde{b}_{1}\left(\delta_{1} \tilde{x}_{1}+\delta_{2} \tilde{x}_{2}-u_{3} E\right)-\Lambda_{3}\left|\tilde{x}_{3}\right|^{1 / 2} \operatorname{sign}\left(\tilde{x}_{3}\right)
\end{aligned}\right.
$$

The sliding mode observer forced the dynamics converge to $s=\tilde{x}_{3}=0 \quad$ sliding surface and evolving (sliding phenomenon) in a dynamic order 2. The objective of the $\Lambda_{3}$ gain is to ensure the attractiveness of the sliding surface. About $\Lambda_{1}$ and $\Lambda_{2}$ they aim to impose the dynamic errors observation on the sliding surface.

\subsubsection{Sliding condition and achievement mode}

The Lyapunov function is positive definite

$$
V(s)=\frac{1}{2} s^{T} s=\frac{1}{2} \tilde{x}_{3}^{2}
$$

The derivative with respect to time is:

$$
\dot{V}(s)=s \cdot \dot{s}=\tilde{x}_{3} \cdot \dot{\tilde{x}}_{3}=\tilde{x}_{3}\left(\Delta f_{3}-\Lambda_{3} \operatorname{sign}\left(\tilde{x}_{3}\right)\right)
$$

According to the direct method of Lyapunov, the $s$ sliding surface is attractive if the derivative is defined:

$$
\dot{V}(s)<0
$$

We have:

$$
\dot{V}(s)<0 \Rightarrow \tilde{x}_{3}\left(\Delta f_{3}-\Lambda_{3} \operatorname{sign}\left(\tilde{x}_{3}\right)\right)<0
$$

Then:

$$
\dot{V}(s)<0 \Rightarrow\left\{\begin{array}{l}
\Lambda_{3}>\Delta f_{3} s i \tilde{x}_{3}>0 \\
\Lambda_{3}>-\Delta f_{3} s i \tilde{x}_{3}<0
\end{array}\right.
$$

This implies that $\forall \tilde{x}_{3}$, it is sufficient that $\Lambda_{3}>\left|\Delta f_{3}\right|_{\max }$ so that $\dot{V}(s)<0$.

If we assume that $\Delta f_{3}$ is bounded, that is to say that observational errors and errors in the system parameters are bounded, then simply check the following condition:

$$
\Lambda_{3}>\left|\Delta f_{3}\right|_{\max }
$$

or

$$
\begin{aligned}
\Lambda_{3} & >\left|\hat{b}_{0} \hat{x}_{3}-b_{0} x_{3}\right|_{\max }+\left|\hat{b}_{1} \hat{x}_{1}-b_{1} x_{1}\right|_{\max } \\
& +\left|\hat{b}_{1} \hat{x}_{2}-b_{1} x_{3}\right|_{\max }+\left|\hat{b}_{1}-b_{1}\right|_{\max }
\end{aligned}
$$

This condition ensures the attractiveness of the sliding surface $s=\tilde{x}_{3}=0$, it depends on committed parametric errors on the load and observation errors. The sliding field is under the $\Re^{3}$ state space where $\tilde{x}_{1}, \tilde{x}_{2}$ verifying condition $\dot{V}(s)<0$.

The observer gains take the form:

$$
\left\{\begin{array}{l}
\Lambda_{1}=-\frac{\Lambda_{3}}{\tau \hat{b}_{1}} \delta_{1} \\
\Lambda_{2}=-\frac{\Lambda_{3}}{\tau \hat{b}_{1}} \delta_{2}
\end{array}\right.
$$

These are interesting alternatives, since they cancel when the $v_{c i}$ associated voltages become unobservable $u_{i+1}-u_{i}=0$. During this time interval, the intermediate voltage observed during the $x_{j=1,2}$ previous command sequence is unchanged.

$\tau$ is a critical parameter that must be precisely adjusted, because it sets the dynamics of the observer during the sliding mode. 


\subsubsection{Simulation results}

Now the results obtained from the operation of the sliding mode observer are presented here.

The value of the observer gain is calculated directly from (23) $\Lambda_{3}=3.5 \cdot 10^{6} \mathrm{~A} / \mathrm{s}$ and it is sufficient to ensure the attractiveness of the sliding surface:

$$
s=\tilde{x}_{3}=0 .
$$

The $\Lambda_{1}$ and $\Lambda_{2}$ gains are set to force the dynamics of the error sliding mode. The theoretical dynamic of the floating capacitor voltage depends on the maximum load current and the value of the floating capacitor:

$$
\frac{d V_{c}}{d t}=\frac{\left|I_{c h}\right|_{\max }}{c}
$$

Taking into account (24), the $\tau$ time constant must be:

$$
\tau=\frac{\Lambda_{3}}{\frac{d V_{c}}{d t} \hat{b}_{1}}
$$

The result of $\Lambda_{1}$ and $\Lambda_{2}$ observer gains are equal to:

$$
\Lambda_{1,2}=-2.10^{6} \delta \mathrm{V} / \mathrm{s} \text {. }
$$

The parameters of the converter are:

$$
\left\{\begin{array}{l}
C_{1}=C_{2}=50 \mu F \\
L=0.5 \mathrm{mH} \\
R=10 \Omega
\end{array}\right.
$$

$E=1,500 \mathrm{~V}$ with $I_{\max }=80 \mathrm{~A}$.

We obtained the following results.

Figure 13 Estimate of the $v_{c 1}$ floating voltage by the super twisting observer (see online version for colours)

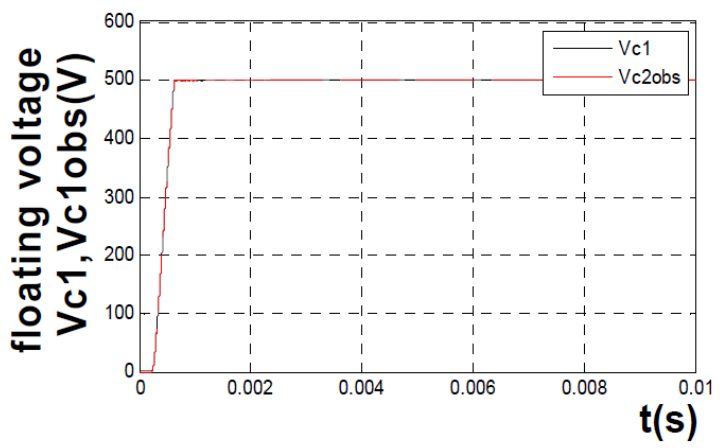

Both Figure 13 and Figure 14 have the observed voltages and the measured voltages across the capacitors. The initial conditions of the observer are zero, apart from transient parts the estimated voltages are very close to the measured voltages.

The graph reveals that the floating voltage across capacitors obtained from the super twisting sliding mode observer is closely matched with those obtained through measurement. Hence, this suggests the viability of use of state estimation concept for series multi-cells converters in real world.
Figure 14 Estimate of the $v_{c 2}$ floating voltage by the super twisting observer (see online version for colours)

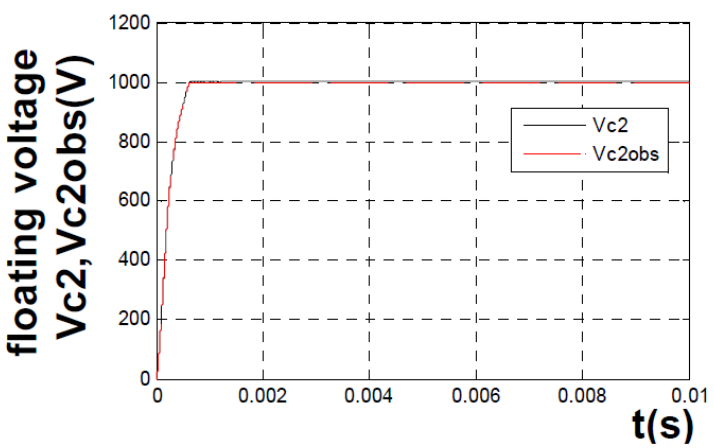

\section{Conclusions}

The paper shows that the multi-cell converters constitute a well-adapted system for the implementation of a higher sliding modes control law.

We then studied the higher order sliding mode observer to the observer based this time on the use of the instant model of the converter, and is to converge to the path of the system on a sliding surface of which the error of current estimate is zero. On this surface, the order of the system is lower, and earnings observations are determined to impose the dynamics of convergence of other variables.

The adopted technique facilitates the generation of the voltages image across capacitors of the converter simply by measuring the current. It is found that the technique possesses the finite time convergence error. The validity of the controller is successfully demonstrated through simulation results for both the mentioned properties of the technique.

Furthermore, authors have reached consensus for realisation of a series multi-cell three-phase converter as future work and also to validate experimentally the results obtained in this paper.

\section{References}

Ben Said, S., Ben Saad, K. and Benrejeb, M. (2013) 'Sliding mode control for a multicell converters', International Conference on Control. Engineering \& Information technology (CEIT'13), Proceedings Engineering \& Technology, No. 2, pp.39-44.

Bensaid, R., Fadel, M. and Meynard, T. (1999) 'Observer design for a three cells chopper using discrete-time model', Electromotion, Vol. 2, No. 2, pp.689-694

Benzineb, O., Taibi, F., Benbouzid, M.E.H., Boucherit, M.S. and Tadjine, M. (2011) 'Multicell converter hybrid sliding mode control', International Review on Modelling and Simulations, Vol. 4, No. 4, pp.1396-1403.

Bestle, D. and Zeitz, M. (1983) 'Canonical form observer design for non-linear time-variable systems', Int. J. Control, Vol. 38, No. 2, pp.419-431

Bethoux, O., Barbot, J.P. and Floquet, T. (2003) Mode glissants et convertisseurs multicellulaires, Cifa, Tunisie. 
Bhave, M., Janardhanan, S. and Dewan, L. (2015) 'A smart higher order sliding mode control of rigid articulated robotic manipulator with passive joints', Int. J. of Modelling, Identification and Control, Vol. 23, No. 3, pp.260-266.

Busawon, K., Farza, M. and Hammouri, H. (1998) 'Observer design for a special class of nonlinear systems', Int. J. Control, Vol. 71, No. 3, pp.405-410

Chen, X. and Zhai, G. (2007) 'Observation for the descriptor systems with disturbances', Nonlinear Dynamics and Systems Theory, Vol. 7, No. 2, pp.121-139.

Dalla Mora, M., Germani, A. and Manes, C.A. (1997) 'State observer for nonlinear dynamical systems', Nonlinear Analysis, Theory, Methods \& Applications, Vol. 30, No. 7, pp.4485-4496.

Dhanalakshmi, K., Umapathy, M. and Ezhilarasi, D. (2015) 'Sliding mode control of shape memory alloy actuated structure for vibration control', Int. J. of Modelling, Identification and Control, Vol. 23, No. 2, pp.121-130.

Donzel, A. (2000) Commande des convertisseurs multiniveaux: Application à un moteur asynchrone, Thèse de doctorat, Institut National Polytechnique de Grenoble

Erickson, R. and Maksimovic, D. (2001) Fundamentals of Power Electronics, 2nd ed., Kluwer Academic Publishers, Dordrecht, The Netherlands.

Gauthier, J.P., Hammouri, H. and Othman, S. (1992) 'A simple observer for nonlinear systems application to bioreactors', IEEE Trans. on Automatic Control, Vol. 37, No. 6, pp. $875-880$.

Ghanes, M., Bejarano, F. and Barbot, J.P. (2009) 'On sliding mode and adaptive observers design for multi cell converter', American Control Conference, USA.

Kaur, S. and Janardhanan, S. (2014) 'Second order sliding mode controller and online path planning for space rovers', Int. J. of Modelling, Identification and Control, Vol. 21, No. 3, pp.321-329.
Kolsi Gdoura, E., Feki, M. and Derbel, N. (2015) 'Sliding mode control of a hydraulic servo system position using adaptive sliding surface and adaptive gain', Int. J. of Systems, Control and Communications, Vol. 23, No. 3, pp.248-259.

Krener, A.J. and Isidori, A. (1983) 'Linearization by output injection and nonlinear observers', Systems \& Control Letters, Vol. 3, No. 1, pp.47-52.

Liu, J., Laghrouche, S., Harmouche, M. and Wack, M. (2014) 'Adaptive-gain second order sliding mode observer design for switching power converters', Control Engineering Practice, Vol. 30, No. 9, pp.124-131

Meradi, S., Benmansour, K., Herizi, K., Tadjine, M. and Boucherit, M.S. (2013) 'Sliding mode and fault tolerant control for multicell converter four quadrants', Electric Power Systems Research, Vol. 95, No. 1, pp.128-139, Elsevier.

Meynard, T., Foch, H., Thomas, P., Courault, J., Jakob, R. and Nahrstaedt, M. (2002) 'Multi-cell converters: basic concepts and industry applications', IEEE Transaction on Industrial Applications, Vol. 49, No. 5, pp.955-964

Röbenack, K. (2007) 'Observer design for a class of nonlinear systems with non-full relative degree', Nonlinear Dynamics and Systems Theory, Vol. 7, No. 4, pp.399-408.

Slotine, J.J.E., Hedrick, J.K. and Misawa, E.A. (1987) 'On sliding observers for nonlinear systems', Journal of Dynamic Systems, Measurement and Control, Vol. 109, No. 3, pp. $245-252$.

Tachon, O., Fadel, M. and Meynard, T. (1997) 'Control of series multicell converters by linear state feedback decoupling', $E P F$, pp.1588-1593, Grenoble.

Zhen, R., Chen, J., Wu, X., Zhu, Q. and Nouri, H. (2014) 'Sliding mode control of uncertain switch systems with time-delay and disturbance', Int. J. of Modelling, Identification and Control, Vol. 21, No. 4, pp.362-369 